\title{
A study of boundary value problem for generalized fractional differential inclusion via endpoint theory for weak contractions
}

\author{
Samiha Belmor ${ }^{1}$, Fahd Jarad ${ }^{2}$, Thabet Abdeljawad ${ }^{3,4,5^{*}}$ (1) and Gülsen Kılınç ${ }^{6}$
}

\author{
"Correspondence: \\ tabdeljawad@psu.edu.sa \\ ${ }^{3}$ Department of Mathematics and \\ General Sciences, Prince Sultan \\ University, P. O. Box 66833, Riyadh \\ 11586, Saudi Arabia \\ ${ }^{4}$ Department of Medical Research, \\ China Medical University, Taichung \\ 40402, Taiwan \\ Full list of author information is \\ available at the end of the article
}

\begin{abstract}
This note is concerned with establishing the existence of solutions to a fractional differential inclusion of a $\psi$-Caputo-type with a nonlocal integral boundary condition. Using the concept of the endpoint theorem for $\varphi$-weak contractive maps, we investigate the existence of solutions to the proposed problem. An example is provided at the end to clarify the theoretical result.
\end{abstract}

MSC: 26A33; 34A60; 30E25

Keywords: Riemann-Liouville fractional derivative with respect to another function; Caputo fractional derivative with respect to another function; Fractional differential inclusion; $\varphi$-Weak contractive

\section{Introduction}

On different time ranges, fractional calculus has had great impact due to a diversity of applications that have contributed to several fields of technical sciences and engineering [1-5]. One of the principal options behind the popularity of the area is that fractionalorder differentiations and integrations are more beneficial tools in expressing real-world matters than the integer-order ones. Various studies in the literature, on distinct fractional operators such as the classical Riemann-Liouville, Caputo, Katugamploa, Hadamard, and Marchaud versions have shown versatility in modeling and control applications across various disciplines. However, such forms of fractional derivatives may not be able to explain the dynamic performance accurately, hence, many authors are found to be sorting out new fractional differentiations and integrations which have a kernel depending upon a function and this makes the range of definition expanded; see [6-8]. Furthermore, models based on these fractional operators provide excellent results to be compared with the integer-order differentiations [9-12].

Recently, the area of fractional-order differential inclusions has become mainly important as these equations were found to be of high importance in modeling stochastic and optimal controls problems [13]. By using techniques of nonlinear analysis the authors stud-

(c) The Author(s) 2020. This article is licensed under a Creative Commons Attribution 4.0 International License, which permits use, sharing, adaptation, distribution and reproduction in any medium or format, as long as you give appropriate credit to the original author(s) and the source, provide a link to the Creative Commons licence, and indicate if changes were made. The images or other third party material in this article are included in the article's Creative Commons licence, unless indicated otherwise in a credit line to the material. If material is not included in the article's Creative Commons licence and your intended use is not permitted by statutory regulation or exceeds the permitted use, you will need to obtain permission directly from the copyright holder. To view a copy of this licence, visit http://creativecommons.org/licenses/by/4.0/. 
ied different aspects such as establishing the existence and the uniqueness of solutions, the upper and lower solutions, and stability. We refer the reader to [14-25] for various qualitative studies.

Details from the historical attitude and recent improvements in the area are detailed in the monograph of Ahmad et al. [26] and the survey of Agarwal et al. [27].

In this paper, we deal with the following $\psi$-fractional differential inclusions:

$$
{ }^{c} \mathcal{D}_{\psi}^{\sigma} u(y) \in Z(y, u(y)), \quad y \in J=[1, T], 1<\sigma \leq 2,
$$

subject to $\psi$-boundary conditions of the form

$$
u(1)=\sum_{i=0}^{m} \lambda_{i} I_{\psi}^{\varrho} h\left(\eta_{i}, u\left(\eta_{i}\right)\right), \quad \delta_{\psi} u(T)=\delta_{\psi} u(1)=0,
$$

where ${ }^{c} \mathcal{D}_{\psi}^{\sigma}$ is the $\psi$-Caputo fractional-order derivative, $Z:[1, T] \times \mathbb{R} \rightarrow \mathcal{P}(\mathbb{R})$ is a multivalued map, $\mathcal{P}(\mathbb{R})$ is the family of all nonempty subsets of $\mathbb{R}, I_{\psi}^{\varrho}$ is the $\psi$-RiemannLiouville fractional integral of order $\varrho>0,0<\eta_{i} \leq T, \delta_{\psi}=\frac{1}{\psi^{\prime}(y)} \frac{d}{d y}, h:[1, T] \times \mathbb{R} \rightarrow \mathbb{R}$ is given continuous function, and $\lambda_{i} \in \mathbb{R}, i=0,1,2, \ldots, m$, are real constants such that

$$
-1<\left(\theta \sum_{i=0}^{m} \lambda_{i} \frac{\left(\psi\left(\eta_{i}\right)\right)^{\varrho}}{\Gamma(\varrho+1)}\right) \leq 0 .
$$

$\theta$ will be determined later. We establish novel existence results of solutions for the above inclusion problem by using the endpoint theorem when the multivalued map is $\varphi$-weak contractive.

The result of the present paper unifies several classes of fractional differential inclusion with different boundary conditions. For example by taking $\psi(y)=y$ in (1.1)-(1.2) the results agree for the classical Caputo fractional inclusions [28] with a combination of classical nonlocal Riemann-Liouville fractional and Neumann boundary conditions of the form:

$$
u(1)=\sum_{i=0}^{m} \lambda_{i} I_{1^{+}}^{\varrho} h\left(\eta_{i}, u\left(\eta_{i}\right)\right), \quad u^{\prime}(T)=u^{\prime}(1)=0,
$$

when $\psi(y)=\ln (y)$, the results agree with the Caputo-Hadamard fractional inclusions [29] equipped with classical fractional integral boundary conditions of Hadamard type of the form

$$
u(1)=\sum_{i=0}^{m} \lambda_{i}{ }^{H} I_{1^{+}}^{\varrho} h\left(\eta_{i}, u\left(\eta_{i}\right)\right), \quad \delta u(T)=\delta u(1)=0,
$$

while the results for generalized Caputo fractional inclusions [30] with nonlocal Katugampola type integral boundary conditions

$$
u(1)=\sum_{i=0}^{m} \lambda_{i}^{\rho} I_{1^{+}}^{\varrho} h\left(\eta_{i}, u\left(\eta_{i}\right)\right), \quad \delta_{\rho} u(T)=\delta_{\rho} u(1)=0,
$$

follow by taking $\psi(y)=y^{\rho} / \rho$. 
The paper is organized as follows. Section 2 recalls some basic and fundamental definitions and lemmas. In Sect. 3, we prove the existence of a solution to the proposed problem (1.1)-(1.2). An example is provided to demonstrate the main results in Sect. 4.

\section{Preliminary results}

Let $\mathcal{W}=C([1, T], \mathbb{R})$ be the set of all continuous functions $u$ from $[1, T]$ into $\mathbb{R}$ with the uniform norm

$$
\|u\|=\sup _{y \in[1, T]}|u(y)| .
$$

$L^{1}([1, T], \mathbb{R})$ be the Banach space of measurable functions $u:[1, T] \rightarrow \mathbb{R}$ with the norm

$$
\|u\|_{1}=\int_{1}^{T}|u(y)| d y .
$$

We define $\mathrm{AC}_{\psi}^{m}([1, T], \mathbb{R})$ by

$$
\operatorname{AC}_{\psi}^{m}([1, T], \mathbb{R})=\left\{u:[1, T] \rightarrow \mathbb{R} ;\left(\delta_{\psi}^{m-1} u\right)(y) \in \mathrm{AC}([1, T], \mathbb{R}), \delta_{\psi}=\frac{1}{\psi^{\prime}(y)} \frac{d}{d y}\right\}
$$

which is supplied with the norm described by

$$
\|u\|_{C_{\psi}^{m}}=\sum_{j=0}^{m-1}\left\|\delta_{\psi}^{j} u(y)\right\|_{\infty}
$$

where $\psi \in C^{m}([1, T], \mathbb{R})$, with $\psi^{\prime}(y)>0$ on $[1, T]$, and

$$
\delta_{\psi}^{j}=\underbrace{\delta_{\psi} \delta_{\psi} \ldots \delta_{\psi}}_{j \text { times }}
$$

and $\mathrm{AC}([1, T], \mathbb{R})$ is the space of absolutely continuous functions from $[1, T]$ into $\mathbb{R}$.

Now we introduce some notations and definitions of fractional calculus with respect to another function and give preliminary results that we will need in our proofs later.

Definition 2.1 ([28]) The $\psi$-fractional integration operator in the Riemann-Liouville sense of order $\sigma>0$ with lower limit 1 for an integrable function $g$ is defined by

$$
I_{\psi}^{\sigma} g(y)=\Gamma(\sigma)^{-1} \int_{1}^{y} \psi^{\prime}(\xi)(\psi(y)-\psi(\xi))^{\sigma-1} g(\xi) d \xi
$$

provided the integral exists. 
Definition 2.2 ([7]) The $\psi$-fractional differentiation operator in the Riemann-Liouville sense of order $\sigma>0$ of a function $g \in \operatorname{AC}_{\psi}^{m}([1, T], \mathbb{R})$ is defined by

$$
\begin{aligned}
\mathcal{D}_{\psi}^{\sigma} g(y)= & I_{\psi}^{m-\sigma}\left(\delta_{\psi}^{m} g\right)(y)+\sum_{j=0}^{m-1} \frac{\left(\delta_{\psi}^{j} g\right)(1)}{\Gamma(j-\sigma+1)}(\psi(y)-\psi(1))^{j-\sigma} \\
= & \Gamma(m-\sigma)^{-1} \int_{1}^{y} \psi^{\prime}(\xi)(\psi(y)-\psi(\xi))^{m-\sigma-1} \delta_{\psi}^{m} g(\xi) d \xi \\
& +\sum_{j=0}^{m-1} \frac{\left(\delta_{\psi}^{j} g\right)(1)}{\Gamma(j-\sigma+1)}(\psi(y)-\psi(1))^{j-\sigma},
\end{aligned}
$$

provided the integral exists, where $m=[\sigma]+1$, and $\Gamma$ is the Gamma Euler function.

Definition $2.3([6,7])$ The $\psi$-Caputo differentiation operator of fractional-order $\sigma>0$ for a given $g \in \mathrm{AC}_{\psi}^{m}([1, T], \mathbb{R})$ is given by

$$
\begin{aligned}
{ }^{c} \mathcal{D}_{\psi}^{\sigma} g(y) & =I_{\psi}^{m-\sigma}\left(\delta_{\psi}^{m} g\right)(y) \\
& =\Gamma(m-1)^{-1} \int_{1}^{y} \psi^{\prime}(\xi)(\psi(y)-\psi(\xi))^{m-\sigma-1}\left(\delta_{\psi}^{m} g\right)(\xi) d \xi, \quad m=[\sigma]+1,
\end{aligned}
$$

provided the integral exists. If $\sigma=m \in \mathbb{N}$ we have

$$
{ }^{c} \mathcal{D}_{\psi}^{\sigma} g(y)=\left(\delta_{\psi}^{m} g\right)(y) .
$$

Lemma 2.4 ([7]) For $\sigma>0$ and a given function $g \in \mathrm{AC}_{\psi}^{m}([1, T], \mathbb{R})$, we have

$$
I_{\psi}^{\sigma c} \mathcal{D}_{\psi}^{\sigma} g(y)=g(y)-\sum_{j=0}^{m-1} \frac{\left(\delta_{\psi}^{j} g\right)(1)}{j !}(\psi(y)-\psi(1))^{j} .
$$

Particularly, for $0<\sigma<1$, we obtain

$$
I_{\psi}^{\sigma c} \mathcal{D}_{\psi}^{\sigma} g(y)=g(y)-g(1)
$$

We will investigate the existence of solutions to the problem (1.1)-(1.2) with the help of the following lemma.

Lemma 2.5 Let $\phi:[1, T] \rightarrow \mathbb{R}$ be a continuous function, and $1<\sigma \leq 2$. Then the $\psi$ fractional problem

$$
\begin{aligned}
& { }^{c} \mathcal{D}_{\psi}^{\sigma} u(y)=\phi(y), \quad y \in[1, T], \\
& u(1)=\sum_{i=0}^{m} \lambda_{i} I_{\psi}^{\varrho} h\left(\eta_{i}, u\left(\eta_{i}\right)\right), \quad \delta_{\psi} u(T)=\delta_{\psi} u(1)=0,
\end{aligned}
$$

is solvable, and its solution is given by

$$
u(y)=I_{\psi}^{\sigma} \phi(y)+\sum_{i=0}^{m} \lambda_{i} I_{\psi}^{\varrho} h\left(\eta_{i}, u\left(\eta_{i}\right)\right) .
$$


Proof Performing the $\psi$-Riemann-Liouville fractional integration $I_{\psi}^{\sigma}$ to both sides of (2.4) and making use of Lemma 2.4, we derive

$$
u(y)=\lambda_{1}+\lambda_{2}(\psi(y)-\psi(1))+I_{\psi}^{\sigma} \phi(y)
$$

where $\lambda_{1}, \lambda_{2}$ are real constants. Applying the $\delta_{\psi}$-differentiation in (2.7) the following equation is formulated:

$$
\left(\delta_{\psi} u\right)(y)=\lambda_{2}+I_{\psi}^{\sigma-1} \phi(y)
$$

Using the boundary conditions $\left(\delta_{\psi} u\right)(T)=\left(\delta_{\psi} u\right)(1)=0$ in (2.8), we get $\lambda_{2}=0$, then using the condition $u(1)=\sum_{i=0}^{m} \lambda_{i} I_{\psi}^{e} h\left(\eta_{i}, u\left(\eta_{i}\right)\right)$ in (2.7), after inserting $\lambda_{2}=0$, gives us

$$
\lambda_{1}=\sum_{i=0}^{m} \lambda_{i} I_{\psi}^{\varrho} h\left(\eta_{i}, u\left(\eta_{i}\right)\right) .
$$

Thus by substituting values of $\lambda_{1}$ and $\lambda_{2}$ in (2.7), we get the solution (2.6).

\section{Main results}

We introduce in this section the function class $\Psi$ of all mappings $\varphi: \mathbb{R}^{+} \rightarrow \mathbb{R}^{+}, \varphi^{-1}(0)=$ $\{0\}$, and $\varphi(z)<z$ for all $z>0, \varphi\left(z_{n}\right) \rightarrow 0$ when $z_{n} \rightarrow 0$

Definition 3.1 ([31]) Let $\mathcal{W}$ be a complete space endowed with a metric $\rho$. A multivalued operator $S: \mathcal{W} \rightarrow \mathcal{P}_{\mathrm{cl}, \mathrm{bd}}(\mathcal{W})$ is said to be a $\varphi$-weak contraction if there exists a function $\varphi \in \Psi$, such that

$$
H_{\rho}(S z, S w) \leq \rho(z, w)-\varphi(\rho(z, w))
$$

for each $z, w \in \mathcal{W}$, where $\mathcal{P}_{\text {cl,bd }}(\mathcal{W})$ is a nonempty collection of all closed and bounded subsets of $\mathcal{W}$, and $H_{\rho}(\cdot, \cdot)$ denotes for the Hausdorff metric on $\mathcal{P}_{\text {cl,bd }}$ given as

$$
H_{\rho}(Q, D):=\max \left\{\sup _{q \in Q} \rho(q, D), \sup _{d \in D} \rho(d, Q)\right\}
$$

where $\rho(Q, d)=\inf _{q \in Q} \rho(q, d)$ and $\rho(q, D)=\inf _{d \in D} \rho(q, d)$. We call an element $z \in \mathcal{W}$ a fixed point of $S$, if $z \in S z$, and an endpoint or stationary point if $S z=\{z\}$. The set of all fixed points of $S$ is denoted by Fix $(S)$, and $\operatorname{End}(S)$ stands for the set of all endpoints of $S$. We say that $S$ fulfills the approximate endpoint property if $\inf _{z \in \mathcal{W}} \sup _{w \in S z} \rho(z, w)=0$.

Lemma 3.2 ([31]) Let $\mathcal{W}$ be a complete space endowed with a metric $\rho$, and $S: \mathcal{W} \rightarrow \mathcal{P}_{\mathrm{cl}, \mathrm{bd}}$ be a multivalued $\varphi$-weak contractive. If $S$ verifies the approximate endpoint property, then $S$ has an endpoint. Moreover, we have $\operatorname{End}(S)=\operatorname{Fix}(S)$.

Definition 3.3 A function $u \in \operatorname{AC}_{\psi}^{2}([1, T], \mathbb{R})$ is called a solution of the inclusion problem (1.1) if there exists a function $l \in L^{1}([1, T], \mathbb{R})$ with $l(y) \in Z(y, u(y))$, a.e. $y \in[1, T]$, such that 
$u$ satisfies conditions (1.2) and

$$
u(y)=I_{\psi}^{\sigma} l(y)+\sum_{i=0}^{m} \lambda_{i} I_{\psi}^{e} h\left(\eta_{i}, u\left(\eta_{i}\right)\right), \quad y \in[1, T]
$$

where $\psi \in C^{2}(J, \mathbb{R})$ such that $\psi^{\prime}>0$ on $[1, T]$.

We set an operator $\mathcal{L}: \mathcal{W} \rightarrow \mathcal{P}(\mathcal{W})$ associated with the problem (1.1)-(1.2) as

$$
\mathcal{L}(u):\left\{f \in \mathcal{W}: f(y)=I_{\psi}^{\sigma} l(y)+\sum_{i=0}^{m} \lambda_{i} I_{\psi}^{\varrho} h\left(\eta_{i}, u\left(\eta_{i}\right)\right), l \in S_{Z, u}\right\},
$$

where $S_{Z, u}$ is the set of selections for $Z$ by

$$
S_{Z, u}=\left\{l \in L^{1}([1, T], \mathbb{R}), l(y) \in Z(y, u(y)) \text {, a.e. } y \in[1, T]\right\} .
$$

Theorem 3.4 Let $\varphi \in \Psi$. Assume that the following hypotheses hold:

(H 1) $Z:[1, T] \times \mathbb{R} \rightarrow \mathcal{P}_{\mathrm{cp}}(\mathbb{R})$ is a Carathéodory bounded multivalued map, where $\mathcal{P}_{\mathrm{cp}}(\mathbb{R})$ is the collection of all nonempty compact subsets of $\mathbb{R}$.

(H 2) For $u, \bar{u} \in \mathbb{R}$, we have

$$
H_{d}(Z(y, u), Z(y, \bar{u})) \leq \frac{\Gamma(\sigma+1)}{(\psi(T))^{\sigma}}(|u(y)-\bar{u}(y)|-\varphi(|u(y)-\bar{u}(y)|)) .
$$

(H 3) There exists $0<\theta<1$, such that

$$
|h(y, u)-h(y, \bar{u})| \leq \theta|u-\bar{u}|
$$

If $Z$ verifies the approximate endpoint property, then the inclusion problem (1.1)-(1.2) has a solution on $[1, T]$, provided that

$$
-1<\left(\theta \sum_{i=0}^{m} \lambda_{i} \frac{\left(\psi\left(\eta_{i}\right)\right)^{\varrho}}{\Gamma(\varrho+1)}\right) \leq 0 .
$$

Proof The proof will be given in two steps, where we show that $\mathcal{L}: \mathcal{W} \rightarrow \mathcal{P}(\mathcal{W})$ given in (3.2) has an endpoint.

Step 1: $\mathcal{L}$ is closed multivalued of $\mathcal{P}(\mathcal{W})$.

Let $u_{n} \in \mathcal{W}$ such that $u_{n} \rightarrow u$, and $\left(f_{n}\right)_{n \geq 1} \in \mathcal{L}(u)$ be a sequence such that $f_{n} \rightarrow f^{*}$ whenever $n \rightarrow+\infty$. Then there exists a $l_{n} \in S_{Z, u_{n}}$ such that, for each $y \in[1, T]$, we get

$$
f_{n}(y)=I_{\psi}^{\sigma} l_{n}(y)+\sum_{i=0}^{m} \lambda_{i} I_{\psi}^{\varrho} h\left(\eta_{i}, u_{n}\left(\eta_{i}\right)\right)
$$

Since $Z$ has compact values, the sequence $\left(l_{n}\right)_{n \geq 1}$ has a sub-sequence, still denoted by $\left(l_{n}\right)_{n \geq 1}$, which converges strongly to some $l \in L^{1}([1, T], \mathbb{R})$, and hence $l \in S_{Z, u}$. For every $v \in Z(y, u(y))$, we have

$$
\left|l_{n}(y)-l(y)\right| \leq\left|l_{n}(y)-v\right|+|v-l(y)|
$$


which implies

$$
\left|l_{n}(y)-l(y)\right| \leq H_{d}\left(Z\left(y, u_{n}\right), Z(y, u)\right) \leq \frac{\Gamma(\sigma+1)}{(\psi(T))^{\sigma}}\left(\left\|u_{n}-u\right\|-\varphi\left(\left\|u_{n}-u\right\|\right)\right) .
$$

Since $\left\|u_{n}-u\right\| \rightarrow 0$ then $\varphi\left(\left\|u_{n}-u\right\|\right) \rightarrow 0$ and $h$ is a continuous function then, for each $y \in[1, T]$,

$$
f_{n}(y) \rightarrow f^{*}(y)=I_{\psi}^{\sigma} l(y)+\sum_{i=0}^{m} \lambda_{i} I_{\psi}^{\varrho} h\left(\eta_{i}, u\left(\eta_{i}\right)\right)
$$

So $f^{*} \in \mathcal{L}$ and $\mathcal{L}$ is closed multivalued.

Step 2: $\mathcal{L}$ is $\varphi$-weak contraction multivalued, i.e. for $u, \bar{u} \in \mathcal{W}$, we show

$$
H_{\rho}(\mathcal{L}(u), \mathcal{L}(\bar{u})) \leq\|u-\bar{u}\|-\varphi(\|u-\bar{u}\|) .
$$

Let $u, \bar{u} \in C(J, \mathbb{R})$ and $f_{1} \in \mathcal{L}(u)$. Then, there exists $l_{1}(y) \in \mathcal{S}_{Z, u}$ such that, for each $y \in[1, T]$,

$$
f_{1}(y)=I_{\psi}^{\sigma} l_{1}(y)+\sum_{i=0}^{m} \lambda_{i} I_{\psi}^{\varrho} h\left(\eta_{i}, u\left(\eta_{i}\right)\right) .
$$

From (H 2) it follows that

$$
H_{d}(Z(y, u), Z(y, \bar{u})) \leq \frac{\Gamma(\sigma+1)}{(\psi(T))^{\sigma}}(|u(y)-\bar{u}(y)|-\varphi(|u(y)-\bar{u}(y)|)) .
$$

Thus, there exists $w \in Z(y, \bar{u}(y))$ provided that

$$
\left|l_{1}(y)-w\right| \leq \frac{\Gamma(\sigma+1)}{(\psi(T))^{\sigma}}(|u(y)-\bar{u}(y)|-\varphi(|u(y)-\bar{u}(y)|)), \quad y \in J .
$$

Define $U:[1, T] \rightarrow \mathcal{P}(\mathbb{R})$ given by

$$
U(y)=\left\{w \in \mathbb{R}:\left|l_{1}(y)-w\right| \leq \frac{\Gamma(\sigma+1)}{(\psi(T))^{\sigma}}(|u(y)-\bar{u}(y)|-\varphi(|u(y)-\bar{u}(y)|))\right\} .
$$

Since $U(y) \cap Z(y, \bar{u})$ is measurable, then we can find a measurable selection $l_{2}(y)$ for $U(y) \cap$ $Z(y, \bar{u})$. Thus $l_{2}(y) \in Z(y, \bar{u}(y))$, and, for each $y \in[1, T]$, we have

$$
\left|l_{1}(y)-l_{2}(y)\right| \leq \frac{\Gamma(\sigma+1)}{(\psi(T))^{\sigma}}(|u(y)-\bar{u}(y)|-\varphi(|u(y)-\bar{u}(y)|)) .
$$

We define $f_{2}(y)$ for each $y \in[1, T]$, as follows:

$$
f_{2}(y)=I_{\psi}^{\sigma} l_{2}(y)+\sum_{i=0}^{m} \lambda_{i} I_{\psi}^{\varrho} h\left(\eta_{i}, \bar{u}\left(\eta_{i}\right)\right) .
$$


Then for $y \in[1, T]$

$$
\begin{aligned}
\left|f_{1}(y)-f_{2}(y)\right| \leq & \frac{1}{\Gamma(\sigma)} \int_{1}^{y}\left|(\psi(y)-\psi(\xi))^{\sigma-1} \psi^{\prime}(\xi)\right|\left|l_{1}(\xi)-l_{2}(\xi)\right| d \xi \\
& +\sum_{i=0}^{m} \lambda_{i} I_{\psi}^{\varrho}\left|h\left(\eta_{i}, u\left(\eta_{i}\right)\right)-h\left(\eta_{i}, \bar{u}\left(\eta_{i}\right)\right)\right| \\
\leq & \frac{1}{\Gamma(\sigma)} \int_{1}^{y}\left|(\psi(y)-\psi(\xi))^{\sigma-1} \psi^{\prime}(\xi)\right| d \xi \\
& \times \frac{\Gamma(\sigma+1)}{(\psi(T))^{\sigma}}(|u(y)-\bar{u}(y)|-\varphi(|u(y)-\bar{u}(y)|)) \\
& +\theta \sum_{i=0}^{m} \lambda_{i} \frac{\left(\psi\left(\eta_{i}\right)\right)^{\varrho}}{\Gamma(\varrho+1)}\left|u\left(\eta_{i}\right)-\bar{u}\left(\eta_{i}\right)\right| \\
\leq & \frac{(\psi(T))^{\sigma}}{\Gamma(\sigma+1)} \frac{\Gamma(\sigma+1)}{(\psi(T))^{\sigma}}(\|u-\bar{u}\|-\varphi(\|u-\bar{u}\|) \\
& +\theta \sum_{i=0}^{m} \lambda_{i} \frac{\left(\psi\left(\eta_{i}\right)\right)^{\varrho}}{\Gamma(\varrho+1)}\|u-\bar{u}\| \\
\leq & \|u-\bar{u}\|-\varphi(\|u-\bar{u}\|) .
\end{aligned}
$$

Therefore,

$$
\left\|f_{1}-f_{2}\right\| \leq\|u-\bar{u}\|-\varphi(\|u-\bar{u}\|)
$$

It follows that $H_{\rho}(\mathcal{L}(u), \mathcal{L}(\bar{u})) \leq\|u(y)-\bar{u}(y)\|-\varphi(\|u(y)-\bar{u}(y)\|)$, for all $u, \bar{u} \in \mathcal{W}$. By hypothesis, since the operator $Z$ has an approximate endpoint property, then by Lemma 3.2 $\mathcal{L}$ has an endpoint $u^{*} \in \mathcal{W}$, i.e. $\mathcal{L} u^{*}=\left\{u^{*}\right\}$, which is also a fixed point. Consequently, the problem (1.1)-(1.2) has a solution $u^{*}$ and the proof is now complete.

\section{An example}

Let $\mathcal{W}=C([1, e], \mathbb{R})$ be the space of all continuous functions defined on $[1, e]$ and $u \in \mathcal{W}$. Consider the following fractional BVP of differential inclusion:

$$
\left\{\begin{array}{l}
{ }^{c} \mathcal{D}_{\psi}^{\sigma} u(y) \in Z(y, u(y)), \quad y \in[1, e], 1<\sigma \leq 2, \\
u(1)=\frac{1}{4} I_{\psi}^{\frac{1}{3}} h\left(\frac{3}{4}, u\left(\frac{3}{4}\right)\right)-\frac{2}{3} I_{\psi}^{\frac{1}{3}} h\left(\frac{1}{4}, u\left(\frac{1}{4}\right)\right), \quad \delta_{\psi} u(e)=\delta_{\psi} u(1)=0,
\end{array}\right.
$$

where $h(y, u)=\frac{u}{e^{y}(u+1)}$, and $\psi(y)=y^{3}$. Obviously $\psi$ is differentiable and an increasing function on $[1, e]$ with $\psi^{\prime}(y)=3 y^{2}$, which is a continuous function on $[1, e]$. Here $Z:[1, e] \times \mathbb{R} \rightarrow$ $\mathcal{P}(\mathbb{R})$ is a multivalued map given by

$$
Z(y, u(y))=\left[0, \frac{\sin (u)}{(1+y)}\right]
$$


Selecting $\varphi(x)=\frac{x}{2}$. It is clear that the function $\varphi \in \Psi$, indeed $\varphi(x)<x$ for all $x \in[1, e]$, $\varphi^{-1}(\{0\})=0, \varphi\left(x_{n}\right) \rightarrow 0$ when $x_{n} \rightarrow 0$

$$
\begin{aligned}
H_{\rho}(Z(y, \vartheta), Z(y, \bar{\vartheta})) & \leq\left|\frac{\sin (\vartheta)-\sin (\bar{\vartheta})}{(1+y)}\right| \\
& \leq \frac{1}{2}|\vartheta(y)-\bar{\vartheta}(y)| \\
& <\frac{\Gamma(\sigma+1)}{e^{3}}(\|\vartheta-\bar{\vartheta}\|-\varphi(\|\vartheta-\bar{\vartheta}\|)) .
\end{aligned}
$$

Hence the condition (H 2) holds for $\vartheta, \bar{\vartheta} \in \mathbb{R}$ a.e $\sigma \in(1,2]$. On the other hand, we have

$$
\begin{aligned}
|h(y, \vartheta)-h(y, \bar{\vartheta})| & \leq \frac{1}{e^{y}}\left|\frac{\vartheta}{\vartheta+1}-\frac{\bar{\vartheta}}{\bar{\vartheta}+1}\right| \\
& =\frac{1}{e^{y}} \frac{|\vartheta-\bar{\vartheta}|}{(\vartheta+1)(1+\bar{\vartheta})} \\
& \leq \frac{1}{e}|\vartheta-\bar{\vartheta}| \\
& \leq \theta|\vartheta-\bar{\vartheta}| .
\end{aligned}
$$

Therefore condition (H 3) holds. With the given data, it is found that

$$
\left(1+\theta \sum_{i=0}^{m} \lambda_{i} \frac{\left(\psi\left(\eta_{i}\right)\right)^{\varrho}}{\Gamma(\varrho+1)}\right)=1+e\left(\frac{\sqrt[3]{3}}{16 \Gamma\left(\frac{4}{3}\right)}-\frac{1}{6 \Gamma\left(\frac{4}{3}\right)}\right)
$$

with

$$
-1<e\left(\frac{\sqrt[3]{3}}{16 \Gamma\left(\frac{4}{3}\right)}-\frac{1}{6 \Gamma\left(\frac{4}{3}\right)}\right) \approx-0.2329498<0
$$

We define an operator $\mathcal{L}: \mathcal{W} \rightarrow \mathcal{P}(\mathcal{W})$

$$
\mathcal{L}(u)=\left\{g \in \mathcal{W}: \text { there exists } l \in S_{Z, u}, g(y)=u(y), \text { for all } y \in[1, e]\right\}
$$

where

$$
u(y)=I_{\psi}^{\sigma} l(y)+\sum_{i=0}^{m} \lambda_{i} I_{\psi}^{\varrho} h\left(\eta_{i}, u\left(\eta_{i}\right)\right)
$$

Note that 0 is a unique endpoint of $\mathcal{L}$, i.e. $\mathcal{L}(0)=\{0\}$, which implies that $\sup _{u \in \mathcal{L}(0)}\|u\|=0$, thus $\inf _{u \in \mathcal{W}} \sup _{g \in \mathcal{L}(u)}\|\vartheta-g\|=0$. The operator $\mathcal{L}$ as a consequence has the approximate endpoint property. Therefore all conditions of Theorem 3.4 are satisfied, then the inclusion problem (4.1) has at least one solution on $[1, e]$.

\section{Conclusion}

In the present work, the endpoint theorem for $\varphi$-weak contractive maps was used to establish the existence results of solutions for fractional differential inclusion which involves 
the $\psi$-Caputo fractional derivative. Systems of fractional differential inclusions with the $\psi$-Caputo derivative provide more adaptable models, in the sense that by a proper choice of the function $\psi$, hidden features of real-world phenomena could be extracted. An illustrative example is presented to point out the applicability of our main results. Our results are not only new in the given configuration but also correspond to some new results associated with the specific choice of the function $\psi$ involved in the given problem.

\section{Acknowledgements}

The third author would like to thank Prince Sultan University for funding this work through research group Nonlinear Analysis Methods in Applied Mathematics (NAMAM) group number RG-DES-2017-01-17. 13

\section{Funding}

Not applicable.

\section{Availability of data and materials}

Not applicable.

\section{Competing interests}

There is no conflict of interest among the authors of the paper regarding the publication.

\section{Authors' contributions}

All the authors have made equal contributions in this paper. All authors read and approved the final manuscript.

\section{Author details}

${ }^{1}$ Department of Mathematics, University of Batna 2, 05078 Batna, Algeria. ${ }^{2}$ Department of Mathematics, Çankaya University, 06790 Etimesgut, Ankara, Turkey. ${ }^{3}$ Department of Mathematics and General Sciences, Prince Sultan University, P. O. Box 66833, Riyadh 11586, Saudi Arabia. ${ }^{4}$ Department of Medical Research, China Medical University, Taichung 40402, Taiwan. ${ }^{5}$ Department of Computer Science and Information Engineering, Asia University, Taichung, Taiwan.

${ }^{6}$ Department of Elementary Education, Faculty of Education, Adiyaman University, 02040 Adiyaman, Turkey.

\section{Publisher's Note}

Springer Nature remains neutral with regard to jurisdictional claims in published maps and institutional affiliations.

Received: 7 April 2020 Accepted: 30 June 2020 Published online: 10 July 2020

\section{References}

1. Hilfer, R.: Applications of Fractional Calculus in Physics. World Scientific, Singapore (2000)

2. Belmor, S., Ravichandran, C., Jarad, F.: Nonlinear generalized fractional differential equations with generalized fractional integral conditions. J. Taibah Univ. Sci. 14(1), 114-123 (2020)

3. Sohail, A., Maqbool, K., Ellahi, R.: Stability analysis for fractional-order partial differential equations by means of space spectral time Adams-Bashforth Moulton method. Numer. Methods Partial Differ. Equ. 34(1), 19-29 (2018)

4. Jarad, F., Abdeljawad, T., Hammouch, Z:: On a class of ordinary differential equations in the frame of Atangana-Baleanu fractional derivative. Chaos Solitons Fractals 117, 16-20 (2018)

5. Ahmed, I., Kumam, P., Jarad, F., Borisut, P., Sitthithakerngkiet, K., Ibrahim, A.: Stability analysis for boundary value problems with generalized nonlocal condition via Hilfer-Katugampola fractional derivative. Adv. Differ. Equ. 2020(1), Article ID 225 (2020)

6. Almeida, R.: A Caputo fractional derivative of a function with respect to another function. Commun. Nonlinear Sci. Numer. Simul. 44, 460-481 (2017)

7. Jarad, F., Abdeljawad, T.: Generalized fractional derivatives and Laplace transform. Discrete Contin. Dyn. Syst., Ser. S 13(3), 709-722 (2020). https://doi.org/10.3934/dcdss.2020039

8. Sousa, J., da Vanterler, C., de Oliveira, E.C.: On the $\Psi$-Hilfer fractional derivative. Commun. Nonlinear Sci. Numer. Simul. 60, 72-91 (2018)

9. Ahmed, I., Kumam, P., Shah, K., Borisut, P., Sitthithakerngkiet, K., Demba, M.A.: Stability results for implicit fractional pantograph differential equations via $\phi$-Hilfer fractional derivative with a nonlocal Riemann-Liouville fractional integral condition. Mathematics 8(1), Article ID 94 (2020). https://doi.org/10.3390/math8010094

10. Luo, D., Shah, K., Luo, Z:: On the novel Ulam-Hyers stability for a class of nonlinear $\psi$-Hilfer fractional differential equation with time-varying delays. Mediterr. J. Math. 16(5), Article ID 112 (2019)

11. Harikrishnan, S., Shah, K., Baleanu, D., Kanagarajan, K.: Note on the solution of random differential equations via $\psi$-Hilfer fractional derivative. Adv. Differ. Equ. 2018, Article ID 224 (2018)

12. Borisut, P., Kumam, P., Ahmed, I., Jirakitpuwapat, W.: Existence and uniqueness for $\psi$-Hilfer fractional differential equation with nonlocal multi-point condition. Math. Methods Appl. Sci. (2020). https://doi.org/10.1002/mma.6092

13. Stankovic, S.S.: Stochastic inclusion principle applied to decentralized automatic generation control. Int. J. Control 72(3), 276-288 (1999)

14. Alqudah, M.A., Ravichandran, C., Abdeljawad, T., Valliammal, N.: New results on Caputo fractional-order neutral differential inclusions without compactness. Adv. Differ. Equ. 2019(1), Article ID 528 (2019)

15. Phung, P., Truong, L.: On a fractional differential inclusion with integral boundary conditions in Banach space. Fract. Calc. Appl. Anal. 16(3), 538-558 (2013) 
16. Abbas, S., Benchohra, M., Petrusel, A.: Ulam stability for partial fractional differential inclusions via Picard operators theory. Electron. J. Qual. Theory Differ. Equ. 2014, Article ID 51 (2014)

17. Rezaigia, A., Kelaiaia, S.: Existence results for third-order differential inclusion with three-point boundary value problems. Acta Math. Univ. Comen. 2, 311-318 (2016)

18. Abbas, S., Benchohra, M., Hamani, S., Henderson, J.: Upper and lower solutions method for Caputo-Hadamard fractional differential inclusions. Math. Morav. 23(1), 107-118 (2019)

19. Belmor, S., Jarad, F., Abdeljawad, T., Alqudah, M.A.: On fractional differential inclusion problems involving fractional order derivative with respect to another function. Fractals 20(8), Article ID 2040002 (2020)

20. Khan, A., Shah, K., Kumam, P., Onsod, W.: An $(\alpha, \vartheta)$-admissibility and theorems for fixed points of self-maps. In: Econometrics for Financial Applications, pp. 369-380. Springer, Cham (2018)

21. Iqbal, M., Shah, K., Khan, R.A.: On using coupled fixed-point theorems for mild solutions to coupled system of multipoint boundary value problems of nonlinear fractional hybrid pantograph differential equations. Math. Methods Appl. Sci. (2020). https://doi.org/10.1002/mma.5799

22. Jarad, F., Harikrishnan, S., Shah, K., Kanagarajan, K.: Existence and stability results to a class of fractional random implicit differential equations involving a generalized Hilfer fractional derivative. Discrete Contin. Dyn. Syst., Ser. S 13(3), 723-739 (2020). https://doi.org/10.3934/dcdss.2020040

23. Etemad, S., Rezapour, Sh., Samei, M.E.: On fractional hybrid and non-hybrid multi-term integro-differential inclusions with three-point integral hybrid boundary conditions. Adv. Differ. Equ. 2020, Article ID 161 (2020)

24. Etemad, S., Ntouyas, S.K.: Application of the fixed point theorems on the existence of solutions for $q$-fractional boundary value problems. AIMS Math. 4(3), 997-1018 (2019)

25. Ntouyas, S.K., Etemad, S.: On the existence of solutions for fractional differential inclusions with sum and integral boundary conditions. Appl. Math. Comput. 266, 235-243 (2015)

26. Ahmad, B., Alsaedi, A., Ntouyas, S.K., Tariboon, J.: Hadamard-Type Fractional Differential Equations, Inclusions and Inequalities. Springer, Cham (2017)

27. Agarwal, R.P., Benchohra, M., Hamani, S.: A survey on existence results for boundary value problems of nonlinear fractional differential equations and inclusions. Acta Appl. Math. 109(3), 973-1033 (2010)

28. Samko, S.G., Kilbas, A.A., Marichev, O.I.: Fractional Integrals and Derivatives, Theory and Applications. Gordon \& Breach, Yverdon (1993)

29. Jarad, F., Abdeljawad, T., Baleanu, D.: Caputo-type modification of the Hadamard fractional derivative. Adv. Differ. Equ. 2012, Article ID 142 (2012)

30. Jarad, F., Abdeljawad, T., Baleanu, D.: On the generalized fractional derivatives and their Caputo modification. J. Nonlinear Sci. Appl. 10(5), 2607-2619 (2017)

31. Moradi, S., Khojasteh, F.: Endpoints of $\varphi$-weak and generalized $\varphi$-weak contractive mappings. Filomat 26, 725-732 (2012)

\section{Submit your manuscript to a SpringerOpen ${ }^{\circ}$ journal and benefit from:}

- Convenient online submission

- Rigorous peer review

- Open access: articles freely available online

- High visibility within the field

- Retaining the copyright to your article

Submit your next manuscript at $\boldsymbol{\nabla}$ springeropen.com 